\title{
Change in Heart Rate Variability Indexes due to high driving workload in turning left at the intersection in real road environment
}

\author{
Weiwei Guo ${ }^{1, a}$, Xiaoting Tian ${ }^{1, b}$, Jiyuan $\operatorname{Tan}^{1, c}$ and Li WANG \\ ${ }^{1}$ Beijing Key Lab of Urban Intelligent Traffic Control Technology, North China University of \\ Technology, Beijing, 10014, China. \\ ${ }^{2}$ Beijing Key Lab of Urban Intelligent Traffic Control Technology, North China University of \\ Technology, Beijing, 10014, China.
}

acynthiaww@foxmail.com, ${ }^{b}$ tianxiaoting625@163.com, ${ }^{c}$ tjyphilip@163.com

Keywords: HRV, driving workload, turning left, RMSSD, PNN50 and LF/HF.

\begin{abstract}
The paper intends to investigate the changes of Heart Rate Variability (HRV) indexes of healthy drivers while driving on a real urban road environment in order to excavate the variation of drivers' driving workload when turning left. Electrocardiograph (ECG) was recorded continuously while drivers driving on a familiar road. The data from three drives were collected for analyzing every fifty minutes. We mainly analyzed the RMSSD, PNN50 and LF/HF of HRV in time-frequency domain which reflects the activity of autonomic nerve and sympathetic nerve. The HRV was extracted from the ECG signal de-noised and filtered. The results show that RMSSD, PNN50 and LF/HF of the driver obviously increased when turning left on the urban road. This reflects that drivers' HRV will be changed significantly with the heavier visual workload. Our findings indicate that physiological signals can provide a metric of driver stress in future cars capable of physiological monitoring.
\end{abstract}

\section{Introduction}

The complexity of urban traffic increases the risk of driver distraction, which increases the driving workload due to the heavier visual task. Workload is the quantity of a person's cognitive capacity necessary to perform a certain task (O' Donnell \& Eggemeier, 1986)[1]. Research about driving workload is mainly focus on the mental workload. According to Yerkes-Dodson's law, while in the high level of mental stress, extremely is easy to cause the driver generate a sense of tension and stress. Accordingly, driver may occurs distraction or error in the aspects of perception and decision making, and eventually lead to the traffic accident[2]. Mental workload under the influence of external traffic environment, individual characteristics (include sex, age..), and the mood of driver while execute the corresponding driving tasks or not related[3]. Effective real-time monitoring of the driver's driving workload can prevent and reduce traffic accidents effectively. Psychophysiology attempts to interpret the psychological processes through their effect on the body state, rather than through task performance or perceptual ratings.

Researchers testified that HRV is a sensitive index for evaluating the mental workload of operator in the field of human factors engineering. Benjamin Eilebrecht[4] studied the relationship between HRV parameters and the mental workload used ECG data acquired from real driving environment. They found that the LF of the HRV obtained by AR transform or FFT transform has better correlation with mental workload. Andreas Henelius[5] shows that the HRV metric can hence be used to distinguish between a low and high mental workload condition during a computerised multitask test. Meshkati, N.[6] (1988) states that heart rate variability is probably the most used physiological measure in workload measurement and references other literature, noting the varied effectiveness of heart rate variability in workload assessment.

Turning left at the intersection, driver need to pay attention to avoid collisions with other vehicles. Mental workload may increase heart rate and decrease heart rate variability at the same time. HRV may be especially useful to collect detailed information of the drowsiness cycle and anticipate risky 
situations while driving. In this paper, we present our analysis based on the time-frequency domain features of HRV. Noelia Rodriguez-Ibañez[7] found that comparing drowsy and alert periods show significant differences $(\mathrm{p}<0.05)$ for SDNN,RMSSD, PNN50, AFS, LF/HF and MEDF.

\section{Methods}

\subsection{Participants}

To reduce inter-subject differences, three male experienced volunteers with more than three years of driving experience were performed the experiments. The participants have no history of cardiovascular disease, and were supervised in order to ensure that they remained adequate sleep. They were prohibited to eat tobacco, alcohol, tea, coffee and other food or medications that may affect the heart rate. Simultaneously, subjects were avoided strenuous exercise before the experiment.

\subsection{Experiment and Data Collection}

The measurements were performed in an ordinary car. The subject was attached to electrodes which were connected to an ambulatory ECG monitoring system, supported by a battery with power supply independence time approximately two hours. In order to meet the test requirements, subjects were required driving on a known route in urban road using a same car. Mitra Taghizadeh-Sarabi[8] introduced a new procedure analyzing turning left and right during driving with constant speed in a pre-designed path. In the experiment, we refer to the above driving scenarios, and varied automotive steering as the independent variable we assumed this will lead to a higher driver workload. For the purpose of the experiment we select a way consisting of several different segments. The ECG data were recorded by ErgoLAB man-machine environment synchronization platform at $512 \mathrm{~Hz}$ on a PC running software.

\subsection{Data Analysis}

The indicators of this work's driving workload analyzing we measured Heart Rate and Heart Rate Variability (HRV), which were generated by ECG. The original ECG signal containing noises includes Power-Line interference, baseline drift and Electromyographical interference. The R-peaks could obtained from the ECG signal, which filtered using Wavelet transform. RR interval is calculated through the R-peaks sequence, and it is the basis of the analysis of Heart Rate Variability. Analyzed time domain indexes were the average Heartbeat interval (AVNN), the value of average heart rate (AVHR). the standard deviation of the time series (SDNN), the standard deviation of the differentiated time series (RMSDD), the adjacent RR interval standard deviation percentage greater than 50ms (PNN50). Frequency domain index is mainly focused on LF/HF.

AVNN and AVHR is calculated by(1), (2),

$$
\mathrm{AVNN}=\frac{1}{N} \sum_{i=1}^{N} R R_{i}
$$

Where $R R_{\mathrm{i}}$ is $\mathrm{i}$-th $\mathrm{RR}$ interval, and $\mathrm{N}$ is the number of the total $\mathrm{RR}$ interval for analysis.

$$
A V H R=\frac{60 * f *\left(\text { num }_{-} R\right)}{R_{n}-R_{1}}
$$

Where num $R$ is the number of R-peaks, $R_{n}$ is the position of the $\mathrm{n}$-th R-peak.

Time domain indexes of HRV is calculated by(3), (4).

$$
\operatorname{SDNN}=\sqrt{\frac{1}{N} \cdot \sum_{i=1}^{N}\left(R R_{i}-\overline{R R}\right)^{2}}
$$

Where $\overline{R R}$ is the average value of RR interval sequence.

$$
\operatorname{RMSSD}=\sqrt{\frac{1}{N-1} \sum_{i=1}^{N-1}\left(R R_{i+1}-R R_{i}\right)^{2}}
$$


Frequency domain analysis of HRV using the modern power spectrum estimation method.AR model.

The three experimental objects' HRV data were analyzed using the statistical software SPSS. Both time domain and frequency domain indexes were considered to explore the HRV changes due to different driving path. The following Tablel is the time-frequency domain indexes of HRV while turning left, Table 2 is in the general straight road.

Table 1 Analysis of HRV index in turning left

\begin{tabular}{|c|c|c|c|}
\hline Index object & Object 1 & Object 2 & Object 3 \\
\hline AVNN(ms) & 772080 & 779.22 & 759.92 \\
\hline AVHR(bpm) & 78 & 77 & 79 \\
\hline SDNN(ms) & 60.08 & 55.35 & 48.07 \\
\hline RMSSD(ms) & 31.86 & 34.41 & 30.81 \\
\hline PNN50(\%) & 13.85 & 12.50 & 10.29 \\
\hline LF/HF(\%) & 22.58 & 21.34 & 17.38 \\
\hline
\end{tabular}

Table 2 Analysis of HRV index in straight road

\begin{tabular}{|c|c|c|c|}
\hline Index object & Object 1 & Object 2 & Object 3 \\
\hline AVNN(ms) & 780.68 & 772.17 & 779.22 \\
\hline AVHR(bpm) & 77 & 78 & 77 \\
\hline SDNN(ms) & 40.28 & 52.01 & 50.23 \\
\hline RMSSD(ms) & 28.04 & 25.42 & 21.69 \\
\hline PNN50(\%) & 7.25 & 5.48 & 3.95 \\
\hline LF/HF(\%) & 13.76 & 2.36 & 10.49 \\
\hline
\end{tabular}

\section{Results and discussion}

The results of data analysis show that studying the time domain indexes of HRV while turning left, the RMSSD and PNN50 index have significantly increased when comparing with driving in the straight road. On the other hand, LF/HF also increased. RMSSD and PNN50 reflect the transient change of RR interval, which can be used to evaluate the vagal activity index. The two indexes increased with the increase of vagal tone[9]. LF/HF reflects the dynamic balance of the sympathetic and vagal activity. The value of $\mathrm{LF} / \mathrm{HF}$ represents the sympathetic nerve excitability, which is correlated with mental workload.

\section{Conclusions}

This paper has explored the change of HRV in driving to assess the driving workload by physiological measurement. The result shows that there are not significant differences between driving straight and turning left for all indexes but RMSSD, PNN50, LF/HF. Results suggests that the vagal activity enhanced and the balance of autonomic nervous activity declined with the increased driving workload. Such finding might provide a theoretical basis for the Auxiliary Driving System to alert the driver according to different driving workload. 


\section{Acknowledgements}

This research is partially supported by the National Natural Science Foundation of China (No. 61503007), Beijing Talents Training funding (No.2014000020124G008), Education Committee (PXM2015_014212_000023). The authors gratefully thank anonymous referees for their useful comments and editors for their work.

\section{References}

[1]. ISO 10075-3:2004 Ergonomic principles related to mental workload--Part 3: Principles and requirements concerning methods for measuring and assessing mental workload.

[2]. Yerkes R M, Dodson J D. The relation of strength of stimulus to rapidity of habit - formation[J]. Journal of comparative neurology and psychology, 1908, 18(5): 459-482.

[3]. O'Donnell, R. D., \& Eggemeier, F. T. (1986). Workload assessment methodology. In K. R. Boff, L. Kaufman, and J. Thomas (Eds.), Handbook of Perception and Human Performance: Volume II, Cognitive Processes and Performance. New York: John Wiley.

[4]. Benjamin Eilebrecht, The Relevance of HRV Parameters for Driver Workload Detection in Real World Driving[J]. Computing in Cardiology, 2012, 39, 409-412

[5]. Andreas Henelius, Kati Hirvonen. Mental Workload Classification using Heart Rate Metrics, 31st Annual International Conference of the IEEE EMBS, Minneapolis, Minnesota, USA, September 2-6, 2009

[6]. Meshkati, N. (1988). Heart rate variability and mental workload assessment. Human Mental Workload. P.A. Hancock and N. Meshkati. Amsterdam, NL, Elsevier Science Publishers B.V. (North-Holland): 101-115.

[7]. Noelia Rodriguez-Ibañez, Changes in Heart Rate Variability Indexes due to Drowsiness in Professional Drivers Measured in a Real Environment. Computing in Cardiology 2012; 39:913-916

[8]. Mitra Taghizadeh-Sarabi1, EEG-based analysis of human driving performance in turning left and right using Hopfield neural network[J]. Taghizadeh-Sarabi et al. SpringerPlus 2013, 2:662

[9]. Zhennan gao,zuogong liu . Effect of $\beta$-blockers on heart rate variability in patients with coronary heart disease [J]. Journal of Clinical Cardiology, 13(3):160. 1997

[10]. Mulder, L.J.M., De Waard, D., Brookhuis, K.A. Estimating mental effort using heart rate and heart rate variability. In: Stanton, N., Hedge, A., Hendrick, H.W., Brookhuis, K.A., Salas, E. (Eds.), Handbook of Ergonomics and Human Factors Methods. Taylor \& Francis, London, pp, $201-208.2004$

[11]. YUAN Wei, GUO Ying-shi., Influence of urban road section types on drivers' workload, Journal of Chang'an University (Natural Science Edition), Vol. 34, No. 5, Sept. 2014 